\title{
Human islets expressing HNF1A variant have defective $\beta$ cell transcriptional regulatory networks
}

\author{
Rachana Haliyur, ${ }^{1}$ Xin Tong, ${ }^{1}$ May Sanyoura, ${ }^{2}$ Shristi Shrestha, ${ }^{3}$ Jill Lindner, ${ }^{4}$ Diane C. Saunders, ${ }^{1}$ Radhika Aramandla, ${ }^{4}$ \\ Greg Poffenberger, ${ }^{4}$ Sambra D. Redick, ${ }^{5}$ Rita Bottino, ${ }^{6}$ Nripesh Prasad, ${ }^{3}$ Shawn E. Levy, ${ }^{3}$ Raymond D. Blind, ${ }^{4,7}$ David M. Harlan, ${ }^{5}$ \\ Louis H. Philipson, ${ }^{2}$ Roland W. Stein, ${ }^{1}$ Marcela Brissova, ${ }^{4}$ and Alvin C. Powers ${ }^{1,4,8}$

\begin{abstract}
'Department of Molecular Physiology and Biophysics, Vanderbilt University, Nashville, Tennessee, USA. ²Departments of Medicine and Pediatrics-Endocrinology, Diabetes, and Metabolism, University of Medical Center, Nashville, Tennessee, USA. ${ }^{5}$ Department of Medicine, Diabetes Center of Excellence, University of Massachusetts Medical School, Worcester, Massachusetts, USA. ${ }^{6}$ Institute of Cellular Therapeutics, Allegheny Health Network, Pittsburgh, Pennsylvania, USA. 'Departments of Pharmacology and Biochemistry, Vanderbilt University, Nashville, Tennessee, USA. ${ }^{7}$ Veterans Affairs Tennessee Valley Healthcare System, Nashville, Tennessee, USA
\end{abstract} \\ Chicago, Chicago, Illinois, USA. ${ }^{3}$ HudsonAlpha Institute for Biotechnology, Huntsville, Alabama, USA. ${ }^{4}$ Division of Diabetes, Endocrinology, and Metabolism, Department of Medicine, Vanderbilt University
}

\begin{abstract}
Using an integrated approach to characterize the pancreatic tissue and isolated islets from a 33-year-old with 17 years of type 1 diabetes (T1D), we found that donor islets contained $\beta$ cells without insulitis and lacked glucose-stimulated insulin secretion despite a normal insulin response to cAMP-evoked stimulation. With these unexpected findings for T1D, we sequenced the donor DNA and found a pathogenic heterozygous variant in the gene encoding hepatocyte nuclear factor$1 \alpha$ (HNF1A). In one of the first studies of human pancreatic islets with a disease-causing HNF1A variant associated with the most common form of monogenic diabetes, we found that HNF1A dysfunction leads to insulin-insufficient diabetes reminiscent of T1D by impacting the regulatory processes critical for glucose-stimulated insulin secretion and suggest a rationale for a therapeutic alternative to current treatment.
\end{abstract}

\section{Introduction}

The clinical diagnosis of diabetes, reflected by hyperglycemia, is straightforward; however, identifying the underlying molecular mechanism(s) is often challenging and sometimes not possible. These challenges are further confounded by well-documented heterogeneity of type 2 diabetes and with heterogeneity of type 1 diabetes (T1D) increasingly being recognized $(1,2)$. Partly, this is because the molecular defect(s) for most forms of diabetes is not known and partly because the molecular phenotyping of tissues involved in human diabetes is inadequate and limited. Critical human tissue and cellular samples relevant to diabetes are challenging to collect, sometimes not accessible, or limited by tissue processing that precludes functional analysis and the application of new technologies. For example, technical barriers prevent sampling of the human pancreas, as it cannot be safely and routinely biopsied in living individuals and rapidly undergoes autodigestion postmortem, hindering adequate molecular diagnostic and clinical phenotyping of the human pancreatic islet in all forms of diabetes.

To overcome such limitations, experimental models have been used to discover critical contributions to our understanding of human physiology and disease. However, in several instances, widely used model systems appear limited in their

Conflict of interest: The authors have declared that no conflict of interest exists. License: Copyright 2019, American Society for Clinical Investigation.

Submitted: May 8, 2018; Accepted: October 24, 2018.

Reference information: J Clin Invest. 2019;129(1):246-251.

https://doi.org/10.1172/JCl121994. translation into clinically relevant information and may even inadvertently be misleading. For example, gene expression responses to inflammation in mice appear to have incomplete predictive clinical value and correlate with only a minority of human gene expression changes (3). Similarly, some rodent models of human monogenic diabetes do not fully reflect the altered glucose homeostasis observed in humans. For example, heterozygous mutations in the key pancreatic islet transcription factor hepatocyte nuclear factor-1 $\alpha$ (HNF1A), which causes the most common form of monogenic diabetes, does not mimic the human disease in mouse models, leaving the pathophysiologic effect of HNF1A genetic variants on the human pancreatic islet incompletely understood (4-6).

To overcome these translational barriers and improve preclinical modeling of human disease, renewed emphasis and new approaches to study human tissue have led to the development of collaborative human tissue repositories or accessible databases such as the Network of Pancreatic Organ donors with Diabetes (nPOD), the Genotype Tissue Expression Project (GTEX), and the Human Islet Research Network (HIRN). Studies of human pancreatic islets have illustrated important similarities and differences from rodent islets in endocrine cell composition and arrangement, innervation, vasculature, and function (7-9).

Using infrastructure to study pancreatic islets and tissue from donors with diabetes in conjunction with the donors' deidentified medical records, investigators are working to better understand the changes in the pancreatic islet in diabetes (10-12). In this report, we describe unexpected functional and molecular findings from the pancreas of an individual with the clinical diagnosis 
A

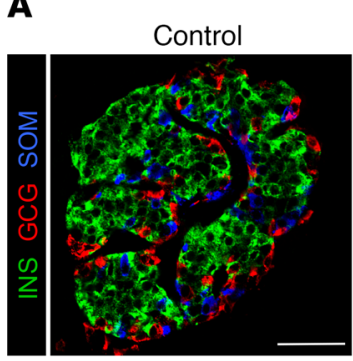

B

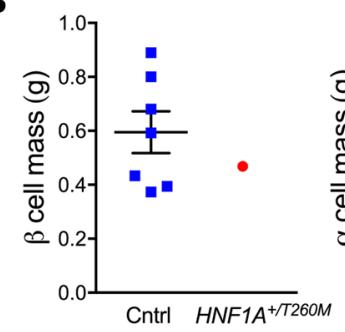

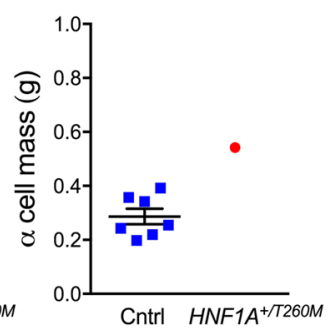
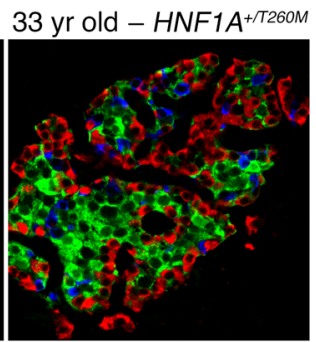

Cntr HNF1A $A^{+/ 260 M}$

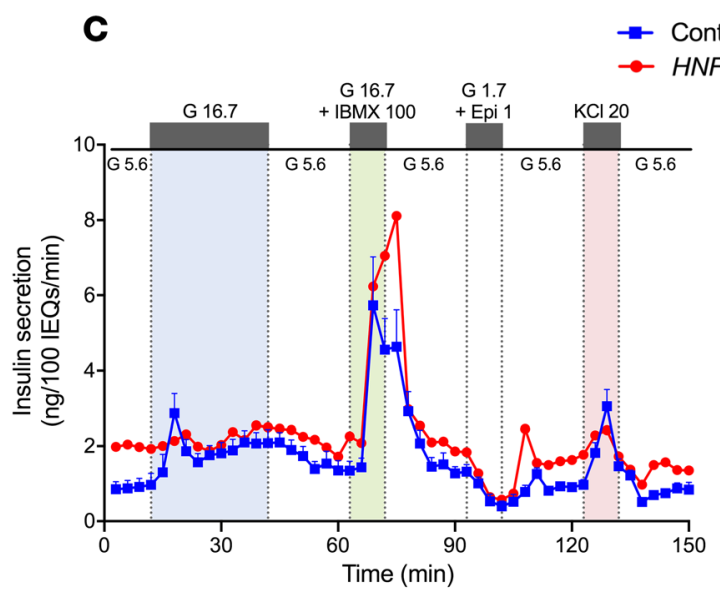

Controls $(n=6)$ HNF1A $^{+/ T 260 M}$

G 16.7
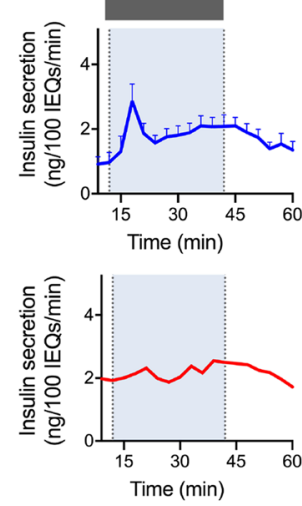

D
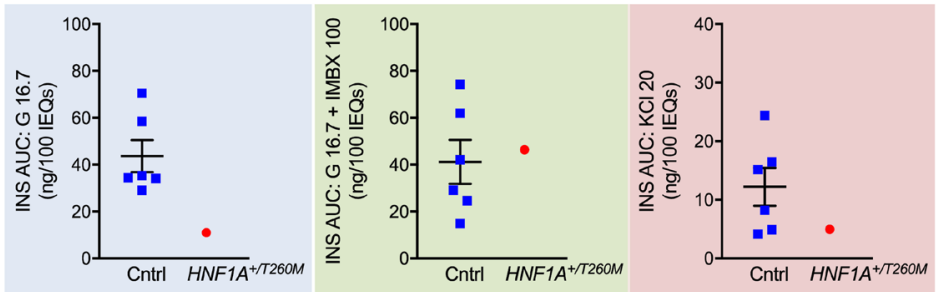

Figure 1. Histological and functional analysis of $\mathbf{H N F 1 A}^{+/ 7260 \mathrm{M}}$ pancreas and islets. (A) Example of expression of insulin (INS), glucagon (GCG), and somatostatin (SOM) in donor's native pancreatic tissue compared with control. Scale bar: $50 \mu \mathrm{m}$. (B) $\beta$ and $\alpha$ cell mass (grams) in HNF1A ${ }^{+/ 7260 M}$ pancreas compared with controls ( $n=7$ donors; ages 10-55 years). Each data point represents the average mass across the combined pancreatic head, body, and tail regions. (C) Insulin secretion in islets isolated from the HNF1A ${ }^{+/ T 260 M}$ pancreas compared with controls ( $n=6$ donors; ages $8-55$ years) and normalized to overall islet cell volume (expressed as islet equivalents, IEQs). Islets were teated with 5.6 mM glucose ( 5 5.6); 16.7 mM glucose ( $(\mathrm{G} 16.7) ; 16.7 \mathrm{mM}$ glucose plus $100 \mu \mathrm{M}$ isobutylmethylxanthine (G 16.7 + IBMX 100); $1.7 \mathrm{mM}$ glucose plus $1 \mu \mathrm{M}$ epinephrine (G 1.7 + Epi 1); or $20 \mathrm{mM}$ potassium chloride (KCI 20) at the indicated times. Insets show average insulin response of controls and HNF1A ${ }^{+/ T 260 M}$ donor to 30 -minute stimulation with $16.7 \mathrm{mM}$ glucose. (D) Integrated insulin secretion was calculated as the area under the curve (AUC) for the indicated secretagogue (shaded to correspond to color-matched regions of perifusion trace in panel $\mathbf{C}$ ). Results of control samples are expressed as mean $\pm \mathrm{SEM}$.

of T1D, thus highlighting how systematic analysis of rare human samples can provide critical insight into human disease and potentially lead to new approaches to therapy.

\section{Results and Discussion}

As part of studies of the pancreas and islets from individuals with T1D (10-12), we were surprised to find that analysis of sections from the head, body, and tail regions of one donor's pancreas showed that all islets contained insulin-positive $\beta$ cells (Figure 1A and Supplemental Figure 1A and insets, and Supplemental Tables 1,3 , and 4 ; supplemental material available online with this article; https://doi.org/10.1172/JCI121994DS1), but lacked insulitis typical of T1D (infiltration of CD45 cells) (13).

Donor pancreas had normal $\beta$ cell mass, but $\beta$ cells were functionally impaired. The donor pancreas had $\beta$ and $\delta$ cell mass within the normal range, with slightly elevated $\alpha$ cell mass (Figure 1B and Supplemental Figure 1B) and an increased $\alpha / \beta$ cell ratio (Supplemental Figure 1, C, G, and H). No $\beta$ cell apoptosis (TUNEL) or proliferation (Ki67) was detected (Supplemental Figure 1D). In a dynamic perifusion system, isolated donor islets had normal insulin content but had higher basal insulin secretion, lacked biphasic glucose-stimulated insulin secretion (GSIS), and had a decreased secretory response to KCl-mediated membrane depolarization (Figure $1 \mathrm{C}$ and inset, Figure 1D, and Supplemental Figure 1G). Despite the lack of GSIS, the donor's islets responded normally to high glucose coupled with the phosphodiesterase inhibitor, isobutylmethylxanthine (IBMX). Moreover, glucagon secretion from donor islet $\alpha$ cells had an abrogated response to potent $\alpha$ cell stimuli such as low glucose $(1.7 \mathrm{mM})$ and epinephrine $(1 \mu \mathrm{M})$ and, strikingly, showed an inhibitory response to membrane depolarization by KCl (Supplemental Figure 1, E, F, and H). Donor pancreas islet innervation and vasculature, important for coordinated islet function in vivo $(8,14)$, were normal (Supplemental Figure 1I). Because of these unexpected histological and functional findings, we sequenced the donor DNA for variants associated with monogenic diabetes and uncovered a heterozygous, disease-associated variant in a conserved region of the $\mathrm{POU}_{\mathrm{H}}$ DNA binding domain of HNF1A (c.779C>T, p.Thr260Met) (15) (Supplemental Table 2 and Supplemental Figure 1J). Variants in HNF1A comprise the most common form of maturity-onset diabetes of the young 3 (termed MODY3) (16).

$H N F 1 A^{T 260 M}$ displayed compromised DNA binding. Nuclear HNF1A protein was detected in both the exocrine and endocrine compartments of the donor pancreas, with normal expression in $\beta$ cells and $\alpha$ cells (Figure 2A and Supplemental Figure 2, A and B). The DNA binding capacity of the altered HNF1A ${ }^{\mathrm{T} 260 \mathrm{M}}$ protein, as assessed by electrophoretic mobility shift assay (EMSA), was severely compromised compared with HNF1A ${ }^{\mathrm{WT}}$ protein (Figure 2B and Supplemental Figure 2, C-E). Accordingly, HNF1A ${ }^{\mathrm{T} 260 \mathrm{M}}$ had little to no ability to stimulate MAFA region 3 enhancer-driv- 
A

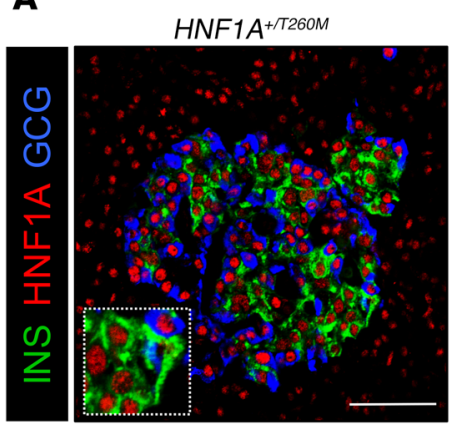

C

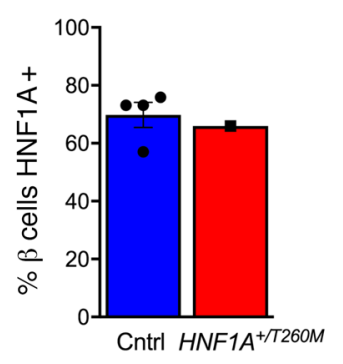

\section{B}

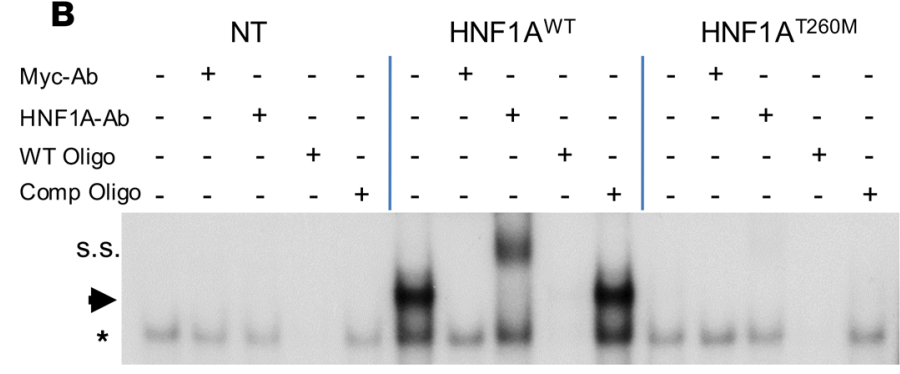

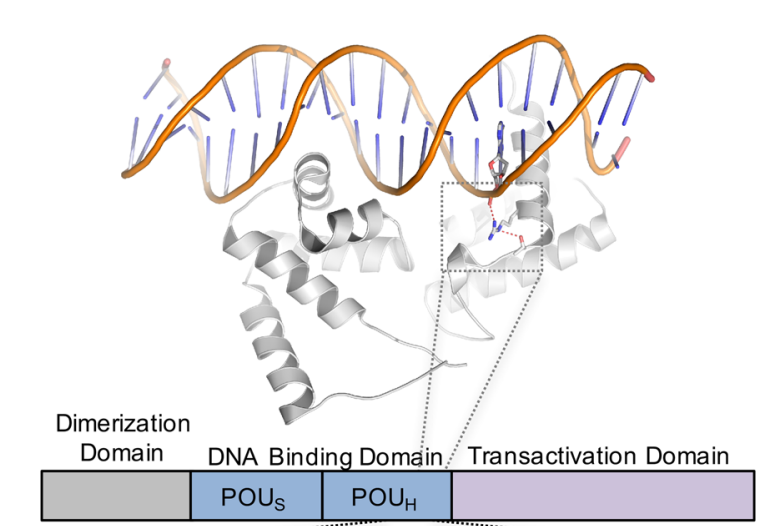

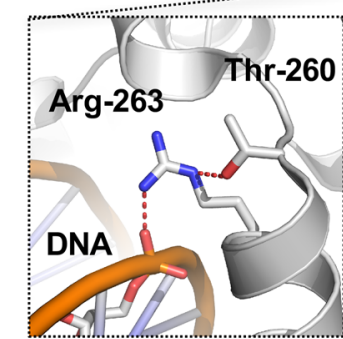

HNF1A ${ }^{W T}$

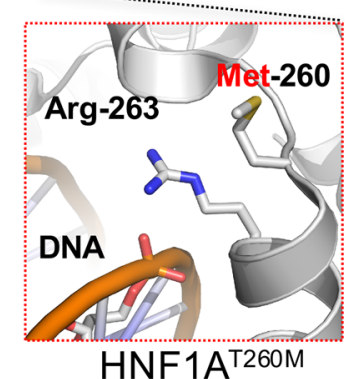

Figure 2. Expression and functional characterization of HNF1A ${ }^{\mathrm{T} 260 \mathrm{M}}$ variant. (A) Analysis of donor's native pancreatic tissue for HNF1A compared with controls ( $n=4$ donors; ages $10-55$ years) revealed HNF1A protein in donor $\beta$ cells. Scale bar: $50 \mu \mathrm{m}$. (B) Electrophoretic mobility shift assay (EMSA) shows that the HNF1A ${ }^{\mathrm{T} 260 \mathrm{M}}$ variant has impaired DNA binding, with loss of the HNF1A-specific DNA binding complex (arrow) in Myc-tagged HNF1A ${ }^{\mathrm{T} 260 \mathrm{M}}$-transfected HeLa cells compared with Myc-tagged HNF1A ${ }^{W T}$. Specificity of this complex (arrow) was shown by exclusive elimination of these species by adding either Myc antibody (Myc-Ab) or unlabeled oligonucleotide (WT Oligo) containing the HNF1A consensus recognition motif, but not a mutated form of this

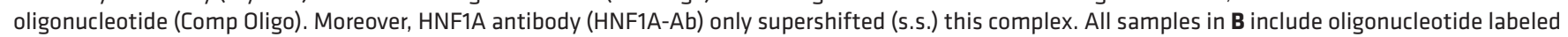
with ${ }^{32} \mathrm{P}$ as described in the supplemental material. Asterisk indicates nonspecific complexes. NT, nontransfected HeLa cells. One representative experiment of 3 is shown. (C) Molecular modeling of the HNF1A ${ }^{\mathrm{T260}}$ variant in PyMOL predicts that the hydroxyl group (red) on threonine 260 (Thr-260) stabilizes arginine 263 (Arg-263) by hydrogen bonding to nitrogen (blue). Arg-263 H-bonds to the DNA backbone of the fifth adenosine of the HNF1A consensus recognition motif ( $5^{\prime}$-CTTCGTTAATAATTCACCACA-3') in control conditions (18). A missense mutation from threonine to methionine at position 260 is predicted to result in the loss of this interaction by destabilizing Arg-263 and subsequently DNA binding. Results of control samples are expressed as mean \pm SEM. See complete unedited blots in the supplemental material.

en reporter activity relative to $\mathrm{HNF} 1 \mathrm{~A}^{\mathrm{WT}}$ in cotransfection assays (Supplemental Figure 2, F and G). Notably, each of these proteins was expressed at similar levels, and WT activation was dependent on HNF1A-site binding, as described previously (17). Furthermore, HNF1A $^{\text {T260M }}$ decreased HNF1A ${ }^{\text {WT }}$ activation in a dose-dependent manner, providing evidence that the dominant-negative action of HNF1A ${ }^{\mathrm{T} 260 \mathrm{M}}$ is due to dimerization with HNF1A ${ }^{\mathrm{WT}}$ (Supplemental Figure 2, F and G). Moreover, protein modeling predicted that disrupted DNA binding results from the missing hydrogen donor at position 260 in the variant protein, which destabilizes the DNA-binding Arg-263 residue (Figure 2C), yet leaves the distinct dimerization domain in the transcription factor intact (18).

$H N F 1 A^{+/ T 260 M} \beta$ cells have preserved markers of $\beta$ cell identity, but changes in processes critical for GSIS. RNA-sequencing and transcriptional profiling of purified $H N F 1 A^{+/ T 260 M} \beta$ cells (Figure 3, A and B) showed relatively preserved expression of INS mRNA and transcription factor markers of $\beta$ cell identity (PDX1, NKX2.2, and NKX6.1), which was confirmed by protein expression analysis (Supplemental Figure 2H). However, decreased expression of other transcription factors associated with mature $\beta$ cell function (i.e., MAFA, SIX3, and RFX6; refs. 17, 19, and 20, respectively) suggests that the $H N F 1 A^{T 260 M}$ variant impacts transcriptional reg- ulatory networks required for $\beta$ cell function rather than maintaining identity. Decreased expression of known (e.g., $M L X I P L$, HNF4A, PKM, OGDH, PPP1R1A, G6PC2, and TMEM27) (21) and previously undescribed HNF1A targets (IAPP, ABCC8, KCNJ11, TMEM37, SYNGR4, and FOXRED2) likely contributes to the loss of GSIS identified by islet perifusion (Figure 3C). Notably, most voltage-gated calcium channels, such as L-type and P/Q-type, were not changed in $H N F 1 A^{+/ T 260 M} \beta$ cells, but ATP-sensitive channels were decreased (ABCC8, KCNJ11, KCNJ8, and FXYD2). Pathway analysis of $H N F 1 A^{+/ T 260 M} \beta$ cells revealed changes in glucose metabolism and ATP production important in glucose-mediated insulin secretory processes as well as in core cellular pathways such as gene transcription, intracellular protein transport (i.e., synthesis, ubiquitination, and exocytosis), cell stress response, and cell signaling (Figure 3D and Supplemental Tables 5 and 7). Approximately $50 \%$ of the genes differentially expressed in $\mathrm{HNF}^{+/ T 260 M}$ $\beta$ cells were also altered in donor $\alpha$ cells (Supplemental Figure 3, A-G), suggesting that HNF1A dysfunction is a common effector in both cell types (Supplemental Tables 5-7). We also noted that other processes such as amino acid nutrient sensing and metabolism, cell cycle regulators, and cell adhesion/motility were altered in $H N F 1 A^{+/ T 260 M}$ islet cells (SLC38A4, GLUL, IGFBP5, and CREB3L1). 
A

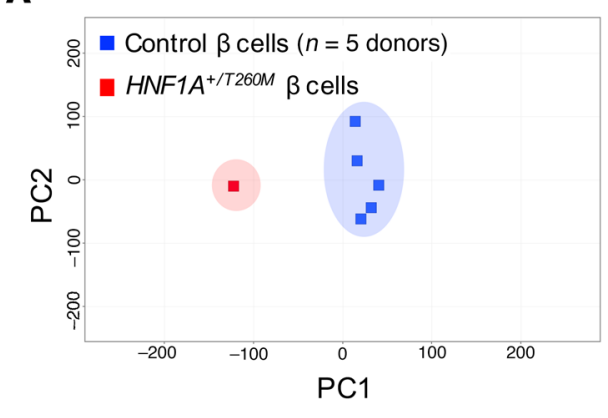

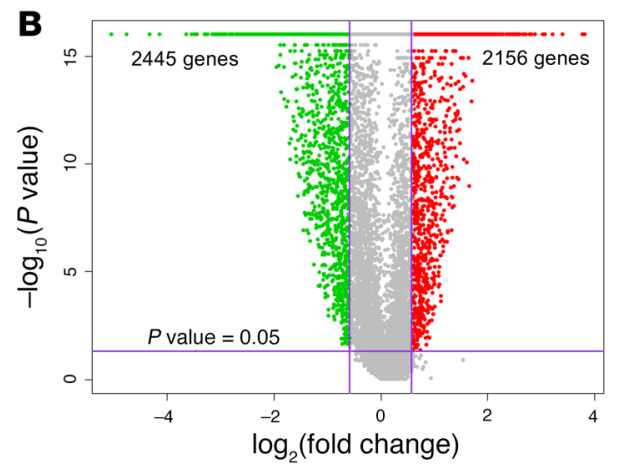

D

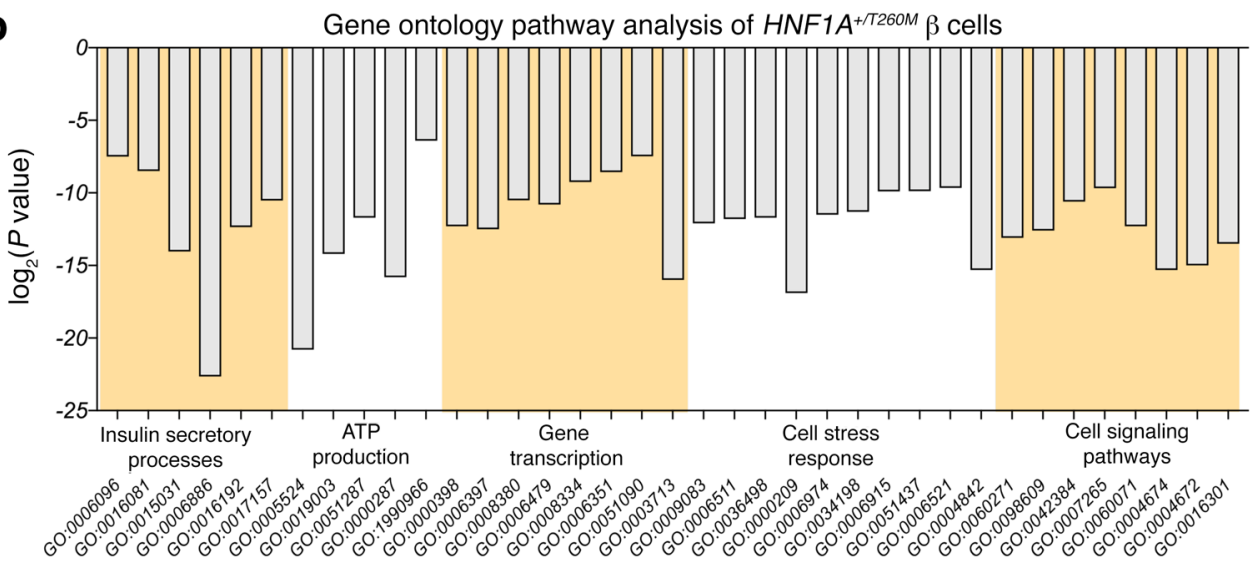

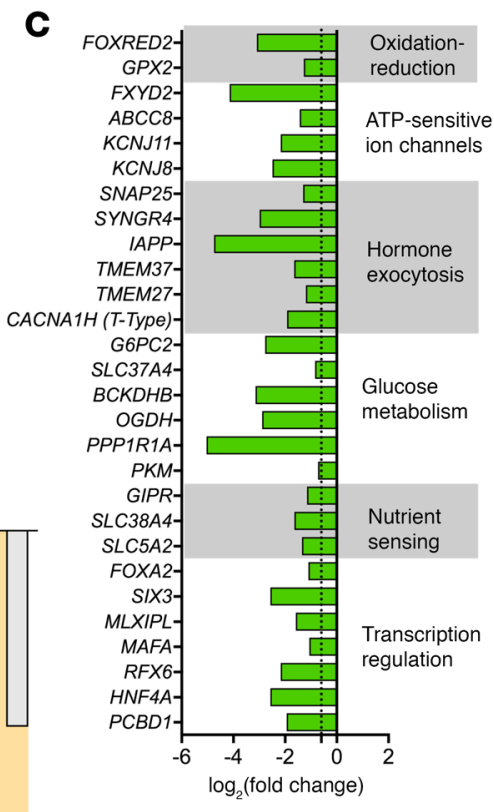

Figure 3. Transcriptomic analysis of HNF1A ${ }^{+/ 2260 M} \boldsymbol{\beta}$ cells. (A) Principal component analysis (PCA) plot depicts clustering of control $\beta$ cells $(n=5$ donors; ages $26-55$ years) (31) separate from HNF1A ${ }^{+/ 7260 M} \beta$ cells. (B) The volcano plot demonstrates transcripts differentially expressed between control and HNF1A ${ }^{+/ T 260 \mathrm{M}} \beta$ cells (red, upregulated gene expression; green, downregulated gene expression). Differential expression was calculated based on fold change (FC) $\geq 1.5$ with a $P$-value cutoff of $<0.05$ for calculated $Z$ score. (C) Genes of interest and HNF1A targets are significantly downregulated in HNF1 ${ }^{+/ T 260 M} \beta$ cells. The vertical dotted line represents $F C=1.5$ times the threshold; $P<0.05$ for all values shown. (D) Significant processes identified by Gene Ontology (GO) term-enrichment analysis are grouped and displayed by their $P$ value on a $\log _{2}$ scale.

This report highlights how molecular and functional findings in unique human samples, even in a single case, can contribute to our understanding of physiology and disease pathogenesis. Levels of HNF1A gene transcript in the human pancreas are substantially lower compared with mouse (22), such that mouse models of heterozygous HNF1A do not phenocopy the human disease (4-6). Missense mutations in the HNF1A dimerization and DNA binding domains account for the majority of described pathogenic HNF1A variants (23). Our modeling predicted that the T260M change would impair DNA binding of HNF1A, rendering this transcriptional factor nonfunctional, which was demonstrated by EMSA analysis. The dose-dependent decrease in transcriptional activity in WT HNF1A-dependent MAFA gene activation by HNF1A ${ }^{\mathrm{T} 260 \mathrm{M}}$ suggests that the dimerization between these proteins leads to impaired DNA binding activity and reduced HNF1A target gene expression in individuals carrying this variant.

In one of the first direct studies of human islets from an individual with a heterozygous, missense variant in the HNF1A locus, we show that this $H N F 1 A^{+/ T 260 M}$ donor had relatively normal $\beta$ cell mass and maintained many key markers of $\beta$ cell identity but lacked an insulin secretory response to glucose challenge. This insulin secretory deficit was accompanied by alterations in genes encoding pathways of glucose metabolism and ATP production, which were also coupled with changes in core metabolic functions, such as gene transcription, protein synthesis and degradation, unfolded protein response, and intracellular and cell-cell communications in $H N F 1 A^{+/ T 260 M} \beta$ cells. From this data set, we propose that this class of loss-of-function variants in HNF1A leads to insulin-insufficient diabetes, not by significant loss of $\beta$ cell mass, but rather by impacting $\beta$ cell transcriptional regulatory networks (HNF4A, MAFA, RFX6, SIX3, FOXA2, and MLXIPL) that results in impairment of $\beta$ cell pathways necessary for a normal insulin response to glucose (Figure 4).

Furthermore, by investigating hormone secretion in isolated pancreatic islets, we discovered that depolarization by $\mathrm{KCl}$, which directly stimulates hormone secretion by activating voltage-dependent calcium channels, was impaired in $H N F 1 A^{+/ T 260 M} \alpha$ and $\beta$ cells, in contrast to results from mouse models (4). Interestingly, elevated basal insulin secretion was observed in islets from this donor, consistent with decreased expression of genes associated with glucose sensitivity of insulin secretion (G6PC2 and SLC37A4) (24). Our data also revealed a previously unrecognized role for HNF1A in $\alpha$ cell function, as HNF1A ${ }^{+/ T 260 M}$ impacted expression of many shared genes involved in hormone-regulated secretion (Supplemental Figure 3C). Transcriptomic analysis also uncovered HNF1A-regulated gene targets in $\beta$ cells, such as PPP1R1A and RFX6, and pathways, like protein synthesis and amino acid metabolism. In addition, a 


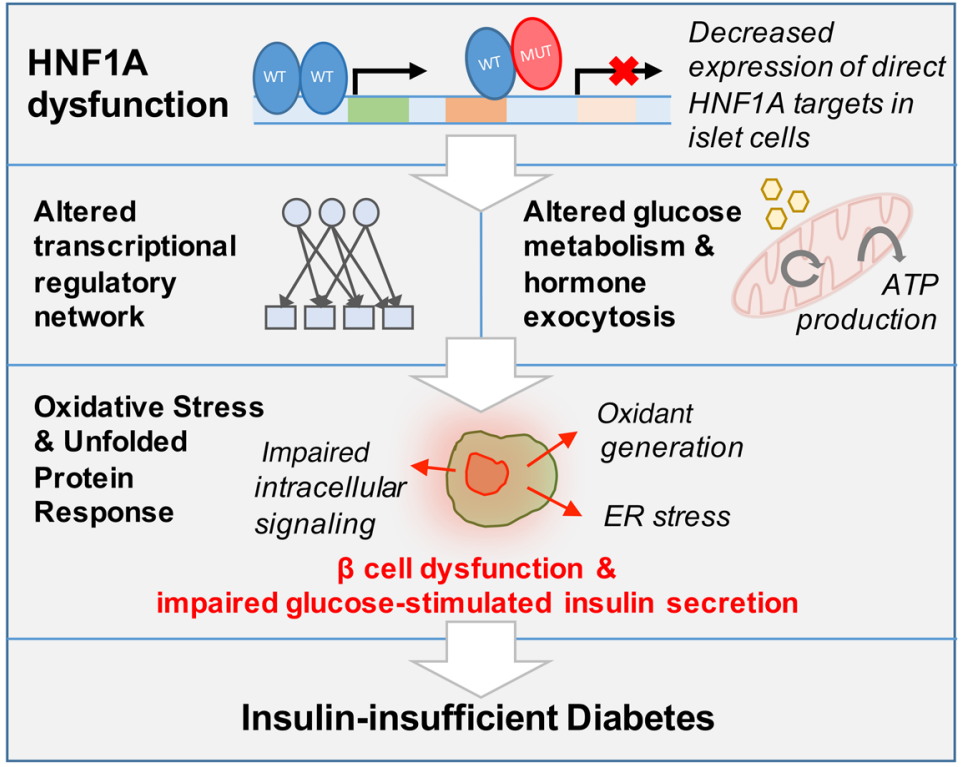

Figure 4. Model of HNF1A dysfunction in human $\beta$ cells. From these results, we propose that dysfunction of HNF1A leads to decreased expression of direct targets, which encompass both enzymatic and gene regulatory products, producing broad changes in transcriptional regulation, glucose metabolism, and hormone secretion. These processes ultimately lead to $\beta$ cell dysfunction and result in clinical manifestation of insulin-insufficient diabetes. significant family history of insulin-deficient diabetes, and/ or a low renal threshold for glucose should prompt genetic testing for HNF1A variants and other monogenic forms of diabetes. Because of this, in collaboration with the Vanderbilt Institutional Review Board (IRB), we are working to communicate our findings to the deidentified donor's family and recommend diagnostic MODY genetic testing in potentially affected family members. Overall, this report shows how integrating clinical information with molecular and cellular analyses identified what appeared to be T1D was in fact part of a broader spectrum of insulin-deficient diabetes and provides translational insight into an incompletely understood form of human diabetes.

\section{Methods}

Detailed methods are in the supplemental materials.

RNA-sequencing data were deposited in the NCBI's Gene Expression Omnibus (GEO) database: GSE106148 (control $\alpha$ cells), GSE116559 (control $\beta$ cells), and GSE120299 ( $H$ NF1A ${ }^{+/ T 260 M}$ donor $\alpha$ and $\beta$ cells).

Statistics. Values are shown as mean \pm standard error of the mean (SEM) for control samples. Data from a sample size of $n=1$ for the donor precluded formal statistical analysis.

Study approval. The Vanderbilt University IRB declared that studies on deidentified human pancreatic specimens do not qualify as human subject research. number of genes differentially regulated in $H N F 1 A^{+/ T 260 M} \beta$ cells included those identified in $\beta$ cell subpopulations by Dorell and colleagues (HCN4, SPP1, KCNJ8, RFX6, SIX3, PPP1R1A, FAM159B, and $G 6 P C 2)$, suggesting that HNF1A may participate in the development of these $\beta$ cell populations (25).

Preserved $\beta$ cell mass in a pancreas with 17 years of MODY3 highlights the importance of clinical identification and intervention even years after the diagnosis of diabetes. Low-dose sulfonylurea therapy produces effective glycemic control in some individuals with MODY3 (26) by stimulating this existing $\beta$ cell reservoir; however, our data provide rationale for a therapeutic alternative to current treatment. Sulfonylureas likely have clinical efficacy by initiating membrane depolarization with potassium channel closure and bypassing effects from impaired ATP production, producing insulin responses comparable to control subjects (27). However, individuals with MODY3 are more sensitive to sulfonylureas (28), which may lead to hypoglycemia and limit this treatment; this might result in part from impaired glucagon secretion related to $\alpha$ cell depolarization (Supplemental Figure 1E). The islet perifusion data from this donor suggest that targeting cAMP-dependent pathways of insulin secretion, such as with glucagon-like peptide 1 (GLP-1) agonists, would have advantages over sulfonylureas, as this pathway of insulin secretion is preserved and accompanied by an intact glucagon response, thus lowering the risk of hypoglycemia in such MODY3 patients (29) (Figure 1C and Supplemental Figure 1E).

Clinical and pathogenic heterogeneity in clinically diagnosed T1D is now increasingly apparent with the ability to study affected human pancreatic tissue $(13,30)$. Clinical features of many MODY phenotypes, which make up $1 \%-5 \%$ of all diabetes cases, can be easily mistaken for T1D. Lack of islet-related humoral autoantibodies,

\section{Author contributions}

$\mathrm{RH}, \mathrm{RWS}, \mathrm{MB}$, and ACP conceived and designed the research. RH, XT, MS, JL, DCS, RA, GP, SDR, and RB performed experiments. RH, XT, MS, SS, RDB, NP, SEL, DMH, LHP, RWS, MB, and ACP analyzed data and interpreted results. $\mathrm{RH}$ prepared figures. RH drafted the manuscript. $\mathrm{RH}, \mathrm{DMH}$, RWS, MB, and ACP edited and revised the manuscript. RH, XT, MS, SS, JL, DCS, RA, GP, SDR, RB, NP, SEL, RDB, DMH, LHP, RWS, MB, and ACP approved the final version of the manuscript.

\section{Acknowledgments}

We thank the organ donors and their families for their invaluable donation and the International Institute for Advancement of Medicine (IIAM), Organ Procurement Organizations, National Disease Research Exchange (NDRI), Integrated Islet Distribution Program (IIDP), and nPOD for their partnership in studies of human pancreatic tissue for research. Tissue from the $H N F 1 A^{+/ T 260 M}$ donor was obtained in collaboration with IIAM. C-peptide was analyzed at Northwest Lipid Metabolism \& Diabetes Research Laboratories and autoantibodies were measured at the Barbara Davis Center for Childhood Diabetes. This research was performed using resources and/or funding provided by the National Institute of Diabetes and Digestive and Kidney Diseases-supported HIRN (RRID:SCR_014393; https:// hirnetwork.org; UC4 DK104211, DK108120, and DK112232), by DK106755, DK72473, DK89572, DK97829, DK94199, DK50203, DK90570, T32GM007347, F30DK112630, and DK20593, and by grants from JDRF, The Leona M. and Harry B. Helmsley Charitable Trust, Department of Veterans Affairs (BX000666), American Cancer Society (RSG-17-063-01), and 
V-Foundation (V2016-015). Flow cytometry analysis was performed by the Vanderbilt Flow Cytometry Shared Resource (P30 CA68485, DK058404).
Address correspondence to: Alvin C. Powers, 7465 MRBIV, 2215 Garland Avenue, Nashville, Tennessee 37323, USA. Phone: 615.936.7678; Email: al.powers@vanderbilt.edu.
1. Hattersley AT, Patel KA. Precision diabetes: learning from monogenic diabetes. Diabetologia. 2017;60(5):769-777.

2. Tuomi T, Santoro N, Caprio S, Cai M, Weng J, Groop L. The many faces of diabetes: a disease with increasing heterogeneity. Lancet. 2014;383(9922):1084-1094.

3. Seok J, et al. Genomic responses in mouse models poorly mimic human inflammatory diseases. Proc Natl Acad Sci U S A. 2013;110(9):3507-3512.

4. Pontoglio M, et al. Defective insulin secretion in hepatocyte nuclear factor 1alpha-deficient mice. J Clin Invest. 1998;101(10):2215-2222.

5. Lee YH, Sauer B, Gonzalez FJ. Laron dwarfism and non-insulin-dependent diabetes mellitus in the Hnf-1alpha knockout mouse. Mol Cell Biol. 1998;18(5):3059-3068.

6. Hagenfeldt-Johansson KA, Herrera PL, Wang $\mathrm{H}$, Gjinovci A, Ishihara $\mathrm{H}$, Wollheim CB. Beta-cell-targeted expression of a dominantnegative hepatocyte nuclear factor- 1 alpha induces a maturity-onset diabetes of the young (MODY)3-like phenotype in transgenic mice. Endocrinology. 2001;142(12):5311-5320.

7. Brissova M, et al. Assessment of human pancreatic islet architecture and composition by laser scanning confocal microscopy. J Histochem Cytochem. 2005;53(9):1087-1097.

8. Rodriguez-Diaz R, et al. Innervation patterns of autonomic axons in the human endocrine pancreas. Cell Metab. 2011;14(1):45-54.

9. Dai C, et al. Islet-enriched gene expression and glucose-induced insulin secretion in human and mouse islets. Diabetologia. 2012;55(3):707-718.

10. Babon JA, et al. Analysis of self-antigen specificity of islet-infiltrating $\mathrm{T}$ cells from human donors with type 1 diabetes. Nat Med. 2016;22(12):1482-1487.

11. Hart NJ, et al. Cystic fibrosis-related diabetes is caused by islet loss and inflammation. JCI Insight. 2018;3(8):e98240.
12. Brissova M, et al. $\alpha$ Cell function and gene expression are compromised in type 1 diabetes. Cell Rep. 2018;22(10):2667-2676

13. Campbell-Thompson $M$, et al. Insulitis and $\beta$-cell mass in the natural history of type 1 diabetes. Diabetes. 2016;65(3):719-731.

14. Brissova M, et al. Pancreatic islet production of vascular endothelial growth factor--a is essential for islet vascularization, revascularization, and function. Diabetes. 2006;55(11):2974-2985.

15. Glucksmann MA, et al. Novel mutations and a mutational hotspot in the MODY3 gene. Diabetes. 1997;46(6):1081-1086.

16. Shields BM, Hicks S, Shepherd MH, Colclough K, Hattersley AT, Ellard S. Maturity-onset diabetes of the young (MODY): how many cases are we missing? Diabetologia. 2010;53(12):2504-2508.

17. Hunter CS, et al. Hnf1 $\alpha$ (MODY3) regulates $\beta$-cell-enriched MafA transcription factor expression. Mol Endocrinol. 2011;25(2):339-347.

18. Chi YI, Frantz JD, Oh BC, Hansen L, Dhe-Paganon S, Shoelson SE. Diabetes mutations delineate an atypical POU domain in HNF-1alpha. Mol Cell. 2002;10(5):1129-1137.

19. Arda HE, et al. Age-dependent pancreatic gene regulation reveals mechanisms governing human $\beta$ cell function. Cell Metab. 2016;23(5):909-920.

20. Chandra V, et al. RFX6 regulates insulin secretion by modulating $\mathrm{Ca}^{2+}$ homeostasis in human $\beta$ cells. Cell Rep. 2014;9(6):2206-2218.

21. Servitja JM, et al. Hnflalpha (MODY3) controls tissue-specific transcriptional programs and exerts opposed effects on cell growth in pancreatic islets and liver. Mol Cell Biol. 2009;29(11):2945-2959.

22. Harries LW, Brown JE, Gloyn AL. Speciesspecific differences in the expression of the HNF1A, HNF1B and HNF4A genes. PLoS ONE. 2009;4(11):e7855

23. Bellanné-Chantelot $\mathrm{C}$, et al. The type and the position of HNF1A mutation modulate age at diagnosis of diabetes in patients with maturityonset diabetes of the young (MODY)-3. Diabetes. 2008;57(2):503-508.

24. Pound LD, et al. G6PC2: a negative regulator of basal glucose-stimulated insulin secretion. Diabetes. 2013;62(5):1547-1556.

25. Dorrell C, et al. Human islets contain four distinct subtypes of $\beta$ cells. Nat Commun. 2016;7:11756.

26. Shepherd M, Shields B, Ellard S, Rubio-Cabezas $\mathrm{O}$, Hattersley AT. A genetic diagnosis of HNF1A diabetes alters treatment and improves glycaemic control in the majority of insulin-treated patients. Diabet Med. 2009;26(4):437-441.

27. Sagen JV, et al. Preserved insulin response to tolbutamide in hepatocyte nuclear factor-1alpha mutation carriers. Diabet Med. 2005;22(4):406-409.

28. Pearson ER, Liddell WG, Shepherd M, Corrall RJ, Hattersley AT. Sensitivity to sulphonylureas in patients with hepatocyte nuclear factor-1alpha gene mutations: evidence for pharmacogenetics in diabetes. Diabet Med. 2000;17(7):543-545.

29. Østoft SH, et al. Glucose-lowering effects and low risk of hypoglycemia in patients with maturity-onset diabetes of the young when treated with a GLP-1 receptor agonist: a double-blind, randomized, crossover trial. Diabetes Care. 2014;37(7):1797-1805.

30. Sanyoura M, et al. Pancreatic histopathology of human monogenic diabetes due to causal variants in KCNJ11, HNF1A, GATA6, and LMNA. JClin Endocrinol Metab. 2018;103(1):35-45.

31. Saunders DC, et al. Ectonucleoside triphosphate diphosphoydrolase-3 antibody targets adult human pancreatic beta-cells for in vitro and in vivo analysis [published online ahead of print November 15, 2018]. Cell Metab. https://doi.org/10.1016/j.cmet.2018.10.007. 